\title{
INTRANIGHT VARIABILITY OF NGC 1275 NUCLEUS IN OPTICS IMPLYING ITS CENTRAL ACTIVITY
}

\author{
I.PRONIK, N.MERKULOVA AND L.METIK \\ Crimean Astrophysical Observatory \\ 334413, p/o Nauchny, Crimea, Ukraine
}

Introduction. NGC 1275 is one of the enigmatic blazars. The nature of central sources of blazars is open to discussion. Variability measurement of continuum is a major touchstone in the understanding of the nature of central sources of AGNs. Below we examine the intranight optical variability of NGC 1275 nucleus using our observations in 1982-1994.

Observations. Two runs of our photoelectric observations were carried out at $1.25-\mathrm{m}$ telescope of Crimean observatory with the time resolution $\sim 4^{m}$ and monitoring during one night - up to 9 hours. In Nov.1982Oct.1987 the photocounting spectrometer was used, diaphragm $\mathrm{D}=10^{\prime \prime}$, $\lambda \sim 5200 \AA, \Delta \lambda=80 \AA$. During 35 nights were made 379 flux measurements. In Dec.1989-Dec.1994 simultaneous UBVRI observations were obtained with the photometer-polarimeter by V.Piirola, using $\mathrm{D}=20^{\prime \prime}, \lambda 3700$ $8300 \AA \AA$. There were 820 flux measurements in each filter during 37 nights.

Light curves. Our observations of NGC 1275 were made during long minimum of the nucleus brightness. The average brightness of the nucleus reduced to $D=5^{\prime \prime}$ decreased from first period to second one up to $40 \%$. Our data do not show the essential systematical trend, but inside the separate nights flux variations were more than $20 \%$. We examine intranight variations using the Structure Function (SF) analysis and the Standard Deviations (SD) of the flux inside the separate nights.

Structure Function analysis. The first order Structure Function is expressed by:

$$
\mathrm{SF}(\Delta \mathrm{t})=<[F(t)-F(t+\Delta t)]^{2}>,
$$

$\mathrm{F}(\mathrm{t})-\mathrm{flux}$ at time t, $\Delta \mathrm{t}-$ time lag between two observations. Variability of AGNs exhibits the processes of noise type, $\mathrm{SF}$ of which is expressed as $\operatorname{SF}(\Delta t)=(\Delta t)^{b}$. Slope $\mathrm{b}=\mathrm{d}[\lg (\mathrm{SF})] / \mathrm{d}[\lg \Delta \mathrm{t}]$ reflects the type of noise processes: if $b=1-$ shot noise, if $b=0$ - flicker noise. $S F$ realization for 
variable flux of NGC 1275 nucleus was made with the application of special program package by Sergeev. We obtained six SFs - one for the first period of observations and five for UBVRI data.

Results. 1. SFs show different slopes " $b$ " for intranight and years variations: $0.23 \leq \mathrm{b} \leq 0.58$ and $0 \leq \mathrm{b} \leq 0.13$ consequently. One can conclude, that there are different processes inside NGC 1275 nucleus: one of them acts at years time scale, another is intranight process.

2. SFs slopes $0.23 \leq b \leq 0.58$ evidences the existence of mixed flickernoise and shot-noise, characteristic time of variations is (3-6) hours.

3. There are negative correlations (SD/ $\sigma-$ Flux) separate for two cases: $\mathrm{SD} \geq 3 \sigma$ (high level variability) and $\mathrm{SD} \leq 3 \sigma$ (low level variability). These data permit to suspect two independent variable components in NGC 1275 nucleus. High level and low level intranight components provide the duty cycle of intranight variations of this nucleus is to be approximately 1 .

4. There was evolution of the process inside NGC 1275 nucleus from 1982-1987 to 1989-1994 for $\lambda \sim 5250 \AA$ : duty cycle of the nucleus for the level of variability $\mathrm{SD} \geq 3 \sigma$ is increased from 0.08 to 0.36 , the slope of SF was increased from $0.23 \pm 0.04$ to $0.36 \pm 0.05$. The contribution of shotnoise to the nucleus' process is increased compare to flicker-noise.

5. There is independent "red activity" of the nucleus: for $\lambda 5300 \AA$ $\mathrm{b}=0.36 \pm 0.0$, duty cycle of high level variability equal to 0.40 and for $\lambda 8300 \AA \mathrm{b}=0.58 \pm 0.05$, duty cycle $=0.53$.

Fitting of the observational data by various models. Two models advanced most frequently for the explanation of the nature of intranight variations in AGNs:

a. Accretion on to black hole. Schwarzshild radius for NGC 1275 nucleus was calculated by Edelson and Malkan (1986) to be equal $2 \cdot 10^{12} \mathrm{~cm}$. One can expect the flux variability of several minutes.

b. Instabilities in shock-in-jet is also possible for the explanation of the intranight variations of NGC 1275 nucleus: obtained parameters of SFs are near to those of 34 radio selected BL Lacertae objects, obtained by Heidt and Wagner (1996). They explained their results by the standard model shocks, propagating down a relativistic jet: shock-in-jet-model.

Intranight red activity of NGC 1275 nucleus independent of optics activity may be associated with "blazar jet". Such component can be suspected for NGC 1275 nucleus from data by Gear et al.(1985) in 1982-1983 in $\mathrm{J}(1.25 \mu \mathrm{m})$ and $\mathrm{H}(1.62 \mu \mathrm{m})$ bands. During 0.5 years the nonstellar component of the flux decayed by a factor more than 4 .

\section{References}

Edelson R.A., Malkan M.A., 1986, ApJ, 308, 59

Heidt J.,Wagner S.J.,1996, A\&A, 305, 42

Gear W.K., Gee G., Robson E.I., Nolt I.G., 1985, MNRAS, 217, 281 